EPJ Web of Conferences 59, 05011 (2013)

DOI: $10.1051 /$ epjconf/20135905011

(C) Owned by the authors, published by EDP Sciences, 2013

\title{
Nonlinear kinetic modeling and simulations of Raman scattering in a two-dimensional geometry
}

\author{
Didier Bénisti ${ }^{1}$, Olivier Morice ${ }^{1, a}$, Laurent $_{\text {Gremillet }}{ }^{1}$, Alexandre Friou ${ }^{1}$ \\ and Erik Lefebvre ${ }^{1}$
}

CEA, DAM, DIF, 91297 Arpajon Cedex, France

\begin{abstract}
In this paper, we present our nonlinear kinetic modeling of stimulated Raman scattering (SRS) by the means of envelope equations, whose coefficients have been derived using a mixture of perturbative and adiabatic calculations. First examples of the numerical resolution of these envelope equations in a two-dimensional homogeneous plasma are given, and the results are compared against those of particle-incell (PIC) simulations. These preliminary comparisons are encouraging since our envelope code provides threshold intensities consistent with those of PIC simulations while requiring computational resources reduced by 4 to 5 orders of magnitude compared to full-kinetic codes.
\end{abstract}

\section{INTRODUCTION}

Predicting Raman reflectivity in fusion devices, such as the future laser Mégajoule (LMJ) or the National Ignition Facility (NIF), is a major issue for inertial confinement fusion, as revealed by recent NIF campaigns [1], but remains a very difficult task. One difficulty to estimate Raman reflectivity is to account for the kinetic effects resulting from the nonlinear modification of the electron distribution function. For small size systems, this may done using full kinetic codes (see for example Ref. [2]). However, the computational resources required by these codes is huge, and it seems quite unlikely that a full kinetic simulation of an actual fusion experiment could be carried out in the near future.

In this paper, we present an alternative approach to full kinetic simulations, based on the use of envelope equations, which do account for nonlinear kinetic effects. The accuracy of our modeling, as regards the collisionless dissipation of the plasma wave, the nonlinear shift of its frequency, or the nonlinear variations of its group velocity, has been tested very carefully against results from Valsov simulations in Refs. [3-5]. Our envelope equations are solved numerically using a C++ code, called BRAMA, which proved to provide reflectivity levels in very good agreement with those computed using a Vlasov code in a one-dimensional geometry, as shown in Ref. [6]. In this article, we present a generalization of our previous equations to a multidimensional geometry, together with preliminary comparisons between PIC and BRAMA simulation results in two dimensions.

\section{THE ENVELOPE EQUATIONS}

Our envelope equations are derived under the assumption that all the waves involved in SRS are quasimonochromatic. The total electric field is thus given by:

$$
\mathbf{E}=E_{p} e^{i \varphi_{p}} \mathbf{u}_{x}+\left[E_{l} e^{i \varphi_{l}}+E_{s} e^{i \varphi_{s}}\right] \mathbf{u}_{y}+\text { c.c. },
$$

\footnotetext{
ae-mail: olivier.morice@cea.fr
}

This is an Open Access article distributed under the terms of the Creative Commons Attribution License 2.0, which permits unrestricted use, distribution, and reproduction in any medium, provided the original work is properly cited. 
where $E_{l}, E_{s}$ and $E_{p}$ are, respectively, the slowly varying amplitude of the laser, scattered and plasma waves, which are supposed to be real and positive; $\varphi_{l}, \varphi_{s}$ and $\varphi_{p}$ are the phases of these waves and $\mathbf{u}_{x}$ and $\mathbf{u}_{y}$ are unitary vectors. Thus, the waves mostly propagate along the $x$ direction. For each wave $\alpha=l, s, p$, we use the eikonal formalism to locally define their frequency and wavevector: $\mathbf{k}_{\alpha}=\nabla \varphi_{\alpha}$ and $\omega_{\alpha}=-\partial_{t} \varphi_{\alpha}$.

SRS-coupled wave envelope equations in a homogeneous two-dimensional plasma then write

$$
\begin{gathered}
\partial_{t} E_{p}+v_{\mathbf{g}_{p}}^{\mathrm{NL}} \partial_{x} E_{p}+\left(v_{\mathbf{g}_{p}}^{\mathrm{NL}} \frac{\delta \mathbf{k}_{p \perp}}{k_{p}}+\mathbf{v}_{\mathbf{g} p \perp}^{\mathrm{NL}}\right) \cdot \nabla_{\perp} E_{p},+v^{\mathrm{NL}} E_{p}=\frac{\Gamma_{p}}{\frac{\partial \chi}{\partial \omega}} E_{l} E_{s}^{*} \\
\partial_{t} E_{s}+\frac{c^{2}}{\omega_{s}}\left[k_{s} \partial_{x} E_{s}-\delta \mathbf{k}_{p \perp} \cdot \nabla_{\perp} E_{s}\right]+i\left[\delta \omega_{p}^{\mathrm{NL}}-v_{\mathrm{g}_{s}} \delta k_{p / /}\right] E_{s}-\frac{i c^{2}}{2 \omega_{s}} \Delta_{\perp} E_{s}=\Gamma_{s} E_{l} E_{p}^{*} \\
\partial_{t} E_{l}+\frac{c^{2} k_{l}}{\omega_{l}} \partial_{x} E_{l}-\frac{i c^{2}}{2 \omega_{l}} \Delta_{\perp} E_{l}=-\Gamma_{l} E_{s} E_{p} .
\end{gathered}
$$

In this system, all coefficients which are superscripted with ${ }^{N L}$ present nonlinear dependence with respect to the plasma wave amplitude, $E_{p}$. These are: the Landau damping rate $v^{\mathrm{NL}}$, the plasma wave group velocity $v_{g p}^{N L}$, the nonlinear frequency shift $\delta \omega_{p}^{\mathrm{NL}}$ and the nonlinear variation of the coupling coefficient $\frac{\partial \chi}{\partial \omega}{ }^{N L}$. As shown in Refs. [3, 6, 7], because the electron motion is nearly adiabatic, these quantities are explicit functions of $E_{p}$, and their values may therefore be tabulated before the resolution of the envelope equations, which saves a lot of computation time. As for the nonlinear wave number shift, $\delta \mathbf{k}_{p}$, it is deduced from the consistency relation $\partial_{t} \delta \mathbf{k}_{p}+\nabla \delta \omega_{p}^{\mathrm{NL}}=0$.

\section{BRAMA SIMULATIONS IN 2D}

All the simulation results presented here are for a $100 \mu \mathrm{m} \times 14 \mu \mathrm{m}$ homogenous two-dimensional plasma irradiated by a laser with wavelength $\lambda=527 \mathrm{~nm}$. The electron density is $N_{e} / N_{c}=0.036$ and the electron temperature is $T_{e}=0.7 \mathrm{keV}$. Thus we have $k_{p} \lambda_{D e} \approx 0.34$. The laser beam is focused at the center of the plasma, where its intensity varies as $\exp \left(-y^{2} / w^{2}\right)$ with a waist $w=1 \mu \mathrm{m}$. Hence, the beam divergence is only 9.5 degrees, which vindicates the use of the paraxial approximation to describe its propagation. The laser peak intensity varies from $2.10^{15}$ to $10^{16} \mathrm{~W} / \mathrm{cm}^{2}$.

For such a homogeneous plasma, the curve $\gamma\left(k_{s}\right)$, plotting the linear SRS growth rate as a function of the scattered wave number exhibits a very sharp maximum at $k_{s}=k_{\max }$. The half width at half maximum of this curve is $\delta k_{s} / k_{s} \approx 3 \%$. Therefore, the scattered wave resulting from SRS should be nearly monochromatic, with $k_{s} \approx k_{\max }$, and this is what we indeed find in our kinetic simulations using the PIC code CALDER [8], where SRS starts from the numerical noise.

For the time being, there is no modeling of plasma fluctuations in BRAMA, and this code actually simulates the optical mixing of the laser with a nearly monochromatic counterpropagating seed whose wave number is $k_{s}=k_{\max }$. The seed intensity has to be chosen small enough to make sure that, in our simulations, we do not miss the linear regime. For this reason, the ratio $\varepsilon$ between the peak laser and seed intensities is chosen to be less than $10^{-5}$. Moreover, as shown in Fig. 1 (a), varying $\varepsilon$ has a limited impact on the laser threshold intensity, $I_{\text {th }}$, and on the SRS reflectivity beyond threshold. Indeed, if we define $I_{\text {th }}$ as the intensity beyond which the SRS reflectivity is larger than $1 \%$, then, when $\varepsilon=10^{-5}$, $I_{\text {th }} \approx 5 \times 10^{5} \mathrm{~W} / \mathrm{cm}^{2}$, while when $\varepsilon=10^{-10}, I_{\text {th }} \approx 7 \times 10^{5} \mathrm{~W} / \mathrm{cm}^{2}$. Hence, a variation in $\varepsilon$ by 5 orders of magnitude induces a change in $I_{\text {th }}$ by less than $50 \%$.

As for the values of SRS-reflectivities actually plotted in Fig. 1 (a), we now need to explain how we estimate them. Fig. 1 (b) shows an example of the time evolution of Raman reflectivity, $R$, calculated with BRAMA when $I_{\text {laser }}=10^{16} \mathrm{~W} / \mathrm{cm}^{2}$ and $\varepsilon=10^{-5}$. In this example, the seed is strongly amplified 
(a)

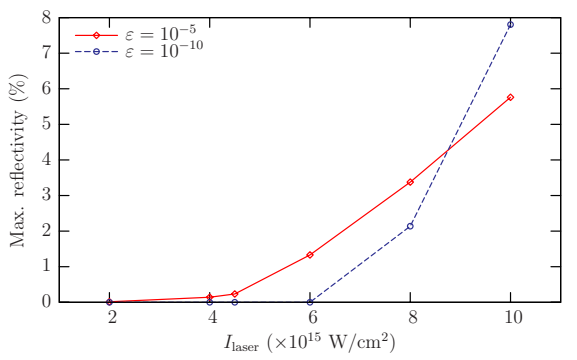

(b)

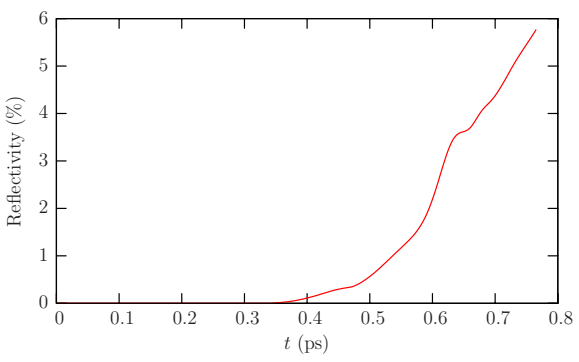

Figure 1. (a) SRS-reflectivity inferred from BRAMA simulations, as a function of the laser intensity. (b) Reflectivity vs time for a BRAMA simulation when $I_{\text {laser }}=10^{16} \mathrm{~W} / \mathrm{cm}^{2}$ and $\varepsilon=10^{-5}$.
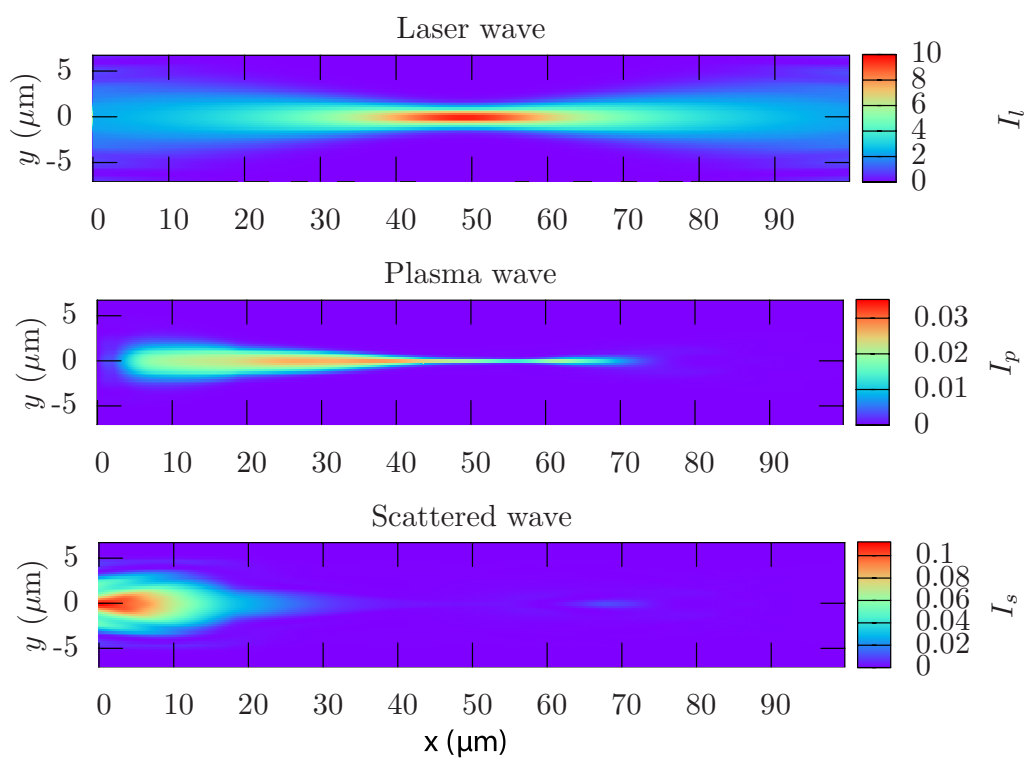

Figure 2. Profiles of the laser, plasma and scatterd waves derived from BRAMA simulations at $t=0.6 \mathrm{ps}$ when $I_{\text {laser }}=10^{16} \mathrm{~W} / \mathrm{cm}^{2}$ and $\varepsilon=10^{-5}$. Plotted data are $I_{\alpha} \equiv 2 \epsilon_{0} c\left|E_{\alpha}\right|^{2}$ in units $10^{15} \mathrm{~W} / \mathrm{cm}^{2}$.

due to Raman scattering and, as $R$ grows, together with the EPW amplitude, we find that this wave undergoes a strong self-focussing, illustrated in Fig. 2. When $R<1 \%$, self-focussing is not yet effective, so that our paraxial approximation is valid. By contrast, when $R$ has reached $6 \%$, the transverse size of the plasma wave packet has become less than the laser wavelength, and we stop the simulation at this point, since this result is unphysical. Actually, although we cannot prove it, it seems quite clear that the strong self-focussing we numerically observe should break the coherence of the three-wave interaction and, therefore, should saturate the growth of Raman scattering. From our BRAMA simulations, we therefore infer that, when $I_{\text {laser }}=10^{16} \mathrm{~W} / \mathrm{cm}^{2}, 1 \%<R<6 \%$, and, in Fig. 1(a), we plot what we believe is an overestimate of Raman reflectivity which should, nevertheless, provide the right order of magnitude for $R$. Direct comparisons, reported in Ref. [9], between results from BRAMA simulations and those obtained in Ref. [2] from PIC simulations show the relevance of our procedure.

We also performed comparisons between PIC and BRAMA simulation results as regards the typical timescale for Raman growth. This is made difficult by the difference in the way SRS is triggered in both codes. In a PIC code, SRS starts from noise and, as a result, the maximum EPW amplitude is always located towards the left-end of the simulation box, because backward SRS naturally tends to grow from 

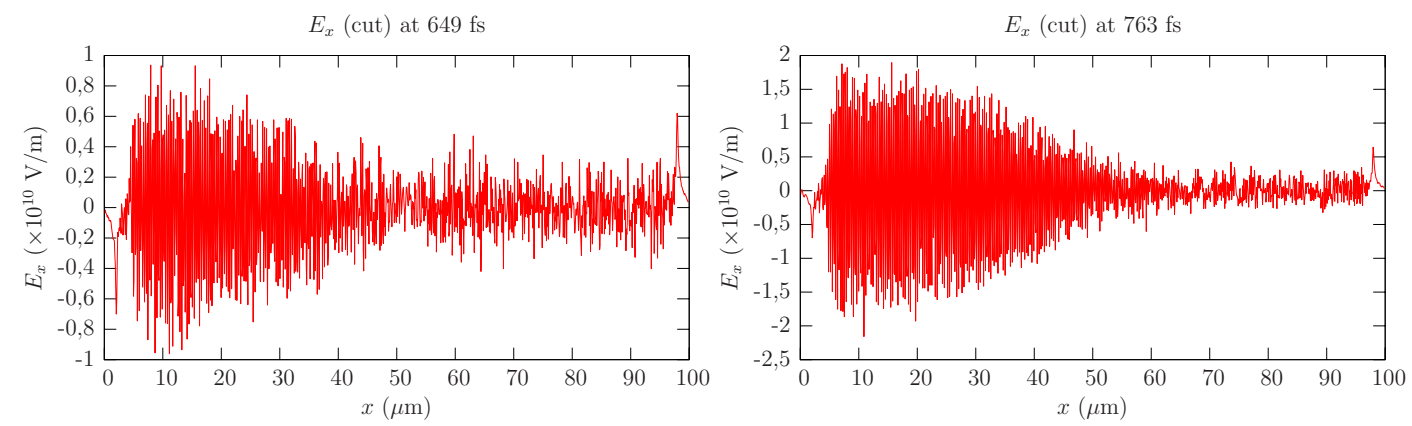

Figure 3. EPW electric field at $y=0$ and at times $t=0.649 \mathrm{ps}$ and $t=0.763$ ps derived from CALDER when $I_{\text {laser }}=10^{16} \mathrm{~W} / \mathrm{cm}^{2}$ and $\varepsilon=10^{-5}$.

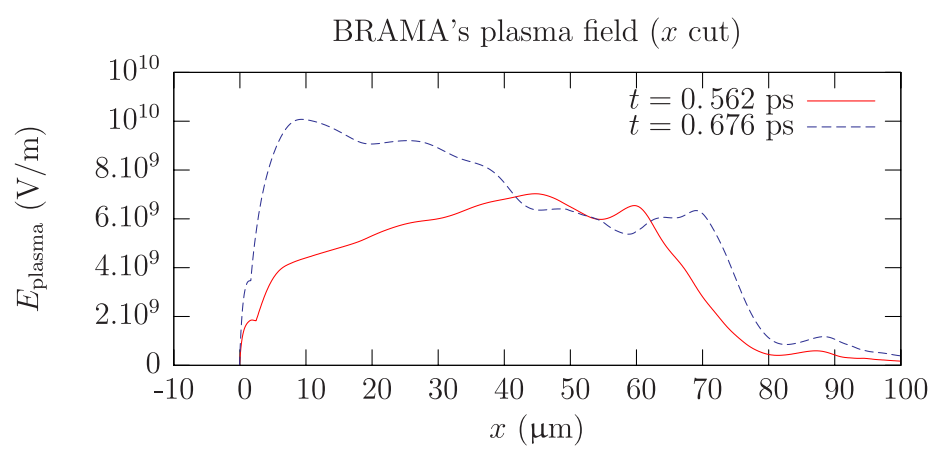

Figure 4. EPW electric field at $y=0$ and at times $t=0.562 \mathrm{ps}$ and $t=0.676 \mathrm{ps}$ derived from BRAMA simulations when $I_{\text {laser }}=10^{16} \mathrm{~W} / \mathrm{cm}^{2}$ and $\varepsilon=10^{-5}$.

right to left. This is clearly illustrated in Fig. 3 obtained with the PIC code CALDER. By contrast, in BRAMA, the EPW starts to grow at the center of the simulation box, where the laser and the seed first meet. Then, as SRS grows, the maximum amplitudes of the plasma and scattered waves move to the left (see Fig. 4). Despite this discrepancy, we can estimate that the rate at which the maximum EPW amplitude grows in CALDER simulations is, from Fig. 3, 1.8.10 $10 / 9.10^{9} /(0.763-0.649) \simeq 17 \mathrm{ps}^{-1}$, while from Fig. 4 we find that, in BRAMA simulations, this rate is $10^{10} / 6.10^{9} /(0.676-0.562)=$ $15 \mathrm{ps}^{-1}$. Hence, the typical timescale for the growth of the EPW in the nonlinear regime is nearly the same in both codes. This shows that these two codes essentially describe the same physics, since the nonlinear kinetic effects mostly affect the growth of the EPW.

\section{CONCLUSION}

In conclusion, based on a direct theoretical calculation of the nonlinear electron motion in a plasma supporting a large amplitude EPW, we derived envelope equations valid in a three-dimensional geometry and accounting for nonlinear kinetic effects. These equations were solved in 2D using our envelope code BRAMA to simulate the optical mixing of the laser with a small amplitude, nearly monochromatic, counterpropagating seed. The simulation results obtained with BRAMA were moreover compared with those of PIC simulations, where SRS starts from noise. Despite this discrepancy, we found a good agreement between the laser threshold intensities, the SRS reflectivities beyond threshold and the typical timescale for Raman growth in the nonlinear regime as given by BRAMA and by a PIC code, while a BRAMA simulation requires a computing time 4 to 5 orders of magnitude shorter than a PIC one. 


\section{IFSA 2011}

\section{References}

[1] D.E. Hinkel et al., Phys. of Plasmas 18, 056312 (2011)

[2] L. Yin et al., Phys. of Plasmas 115, 013109 (2008)

[3] D. Bénisti, D. Strozzi and L. Gremillet, Phys. of Plasmas 15, 030701 (2008)

[4] D. Bénisti et al., Phys. Rev. Lett. 103, 155002 (2009)

[5] D. Bénisti et al., Phys. of Plasmas 17, 082301 (2010)

[6] D. Bénisti et al., Phys. of Plasmas 17, 102311 (2010)

[7] D. Bénisti and L. Gremillet, Phys. of Plasmas 14, 042304 (2007)

[8] E. Lefebvre, N. Cochet, S. Frizler et al., Nucl. Fusion 43, 629 (2003)

[9] D. Bénisti et al., Phys. of Plasmas 19, 056301 (2012) 